\title{
Gender Classification in Human Face Images for Smart Phone Applications Based on Local Texture Information and Evaluated Kullback-Leibler Divergence
}

\author{
Shervan Fekri-Ershad ${ }^{1,2}$ \\ ${ }^{1}$ Faculty of Computer Engineering, Najafabad Branch, Islamic Azad University, Najafabad, Iran \\ ${ }^{2}$ Big Data Research Center, Najafabad Branch, Islamic Azad University, Najafabad, Iran
}

Corresponding Author Email: fekriershad@pco.iaun.ac.ir

https://doi.org/10.18280/ts.360605

Received: 11 September 2019

Accepted: 16 November 2019

\section{Keywords:}

gender classification, human recognition, Improved local binary patterns, facial images, Kullback-Leibler divergence ratio, smart phone applications

\begin{abstract}
One of the main steps in human identification is gender classification which can increase the identification accuracy. In many smart phone applications, human identification plays an important role in different reasons such as login permission, sign up certificates, etc. So, accurate gender classification algorithms may increase the accuracy of smart phone applications and reduce its complexity. Also, one of the benefits of gender classification algorithms is for parents to monitor the social network contacts of their child in terms of gender. Different methods have been proposed to do it accurately so far. In all methods, classification accuracy is the main challenge for researchers. But, in smart phone applications, some challenges such as rotation, gray scale variations may reduce the accuracy. In this respect, a rotation invariant approach is proposed in this paper to classify genders in human face images based on improved version of local binary patters (ILBP). Local binary pattern (LBP) is a texture descriptor, which extract local contrast and spatial structure information. Some issues such as noise sensitivity, rotation sensitivity and low discriminative features can be considered as disadvantages of the basic LBP. ILBP solves the above disadvantages using a new theory for binary patterns categorization. The proposed approach includes two stages. First of all, a feature vector is extracted for human face images based on ILBP. Next, KullbackLeibler divergence classifier is used to classify gender. In this paper Kullback Leibler classifier is evaluated based on log likelihood ratio as distance measure. In the result part, two databases, self-collected and ICPR are used as human face database. Results are compared by different well known methods in this literature that shows the high quality of the proposed approach in terms of accuracy rate. Other main advantages of our approach are rotation invariant, low noise sensitivity, size invariant and low computational complexity. The proposed approach decreases the computational complexity of smartphone applications because of reducing the number of database comparisons. It can also improve performance of the synchronous applications in the smartphones because of memory and CPU usage reduction.
\end{abstract}

\section{INTRODUCTION}

Human's face is a major feature in visual machine learning and image processing systems to identify desired aims. A face carries discriminative and separable information consisting gender, age, ethnicity, etc. [1]. Face information is applicable in many cases such as human-computer interaction, image retrieval, biometric authentication, drivers monitoring, human-robot interaction, sport competition senses analysis, video summarizing, and image/video indexing.

Gender classification may decrease the computational complexity of human identification systems. In near all of the biometric identification systems, a big database is used includes large number of humans, whether man or woman.

There are too many smart phone applications which are used human identification process in many reasons such as login permission, find friends, sign up license, etc. Many smartphone applications usually use the main memory of the mobile to perform the identification. Therefore, reducing the computational complexity of this task will greatly affect the performance of smartphones.

Suppose that in a big database, there are $n$ images from human faces. In a human identification system, the query image should be compared to all of the images inside the database, so that the diagnosis can be done accurately. It means, $n$ times the feature comparison will be needed. Now, if the gender of the query image is clear, it's just that, according to the likelihood rule, the features of the query image will be compared to 50 percent of the images inside the database ( $n / 2$ samples). Therefore, if the computational complexity of the gender classification is less than the computational of comparing the query image with $50 \%$ of the base images, the act of gender classification can greatly increase the performance of the smart phones. This claim shows that, unlike to belief of many researchers, gender classification can be one of the most widely used areas in image processing, with the application in new technologies such as smart phones.

On the other hand, in some of the smartphone applications, gender classification plays an important role. For example, in some cases, it is important for parents to identify the gender of their child's contacts specially in social networks. The process of a human identification system is shown in Fig. 1 based on two different ways in smartphones. As mentioned above, the proposed method can improve mobile performance in either of 
the two modes shown in Figure 1.

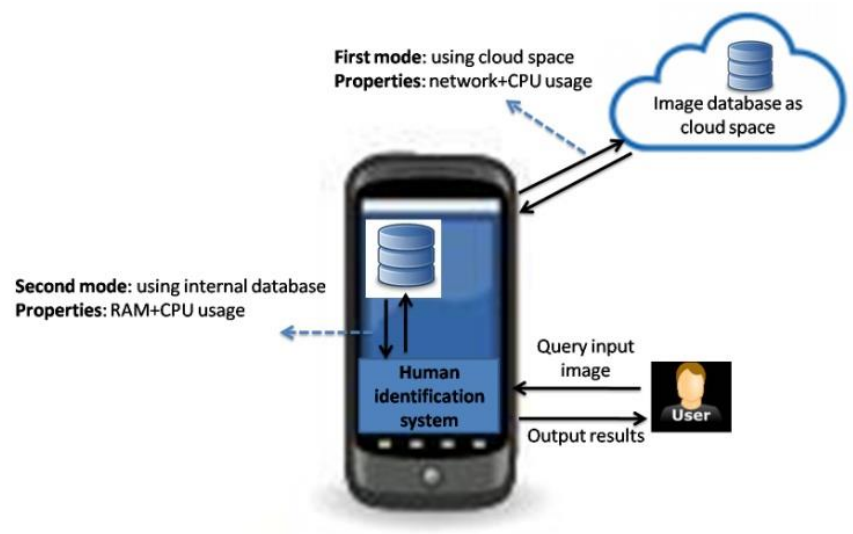

Figure 1. Human identification process in smartphones based on two different ways

In this regard, the main contributions of this paper are as follows:

I. Developing a robust approach for gender classification from human faces with higher accuracy than existing algorithms

II. Propose the idea of using a gender classification algorithm for smart phones from two perspectives as follows:

- Reduce the computational complexity of the human identification applications

- Increase the parental monitoring power over children.

Most of the works in this scope involves two stages: extracting features from faces and classifying those features based on a pre-labeled data. Therefore gender classification approaches can be categorized in terms of feature extraction algorithm or classification methods. The feature extraction algorithms can be broadly grouped into two groups as follows:

I) Appearance-based

II) Geometry-based methods

All of the appearance-based methods try to extract information of the whole image rather than local spatial features. But, in the geometry-based approaches, the local geometric features such as distance between eyes, face width, length, nose size and etc., of a face are considered. The proposed approach extracts local texture information, so it can be categorized as appearance-based approaches. Hence, in the next section, only the works related to appearance-based methods are discussed. For the classification phase, previous works usually used support vector machine, k- nearest neighbors, neural networks, distribution modeling, etc.

Local binary pattern (LBP) is a semi parametric descriptor which describes the local contrast and local spatial structure of an image. LBP commonly is used in computer vision systems to describe image texture. It provides discriminative texture features to identify image content. In this paper an approach is proposed for gender classification of human based on improved version of LBP. First of all, improved LBP is used to describe input human facial image as a feature vector. Next, a classification phase is needed to identify the gender of the test image. In this paper, Kullback-Leibler (KL) divergence algorithm is evaluated based on log-likelihood ratio. So, evaluated $\mathrm{KL}$ is used for classification phase.

\section{RELATED WORKS}

As it was mentioned in previous section, gender classification methods are different in feature extraction and classification algorithms. Briones et al. [2] proposed a multi agent system to classify gender and age in human face images. An innovative method is proposed for image acquisition, preprocessing and final classification [2]. Experimental results show that Fisherfaces method is more effective than multi layer perception (MLP). Also, its training time is shorter because of dimension reducing [2].

Moghaddam et al. [3] used a non-linear version of support vector machine (SVM) to classify faces from thumbnail images with low resolutions. SVM is set up with Gaussian RBF and cubic polynomial kernels [3]. Moghaddam et al. [3] designed different experiments based on other classifiers such as linear discrimination analysis (LDA), RBFs, K-Nearest Neighbor. They used a database with 1,755 thumbnail images including 1044 males and 711 females. Best accuracy was obtained using SVM along with Gaussian RBF kernel with error rate of 3.38 percent for males and 2.05 percent for females. Regardless of high detection rate, this method has some disadvantages such as: collecting a database of thumbnails is not possible in many cases, the computational complexity is very high because of evaluation and using RBF kernel [3].

Many other human properties are discussed in papers until now. For instance, Matta et al. [4] combined spatial information of human parts such as head motion, mouth motion, and facial appearance to extract gender features. Yang et al. [5] developed a fully automatic learner system for combined face detection and gender classification. They used maximum-likelihood estimation for face detection and for facial feature detection.

Shakhnarovich, et al. [6] applied AdaBoost to the features used by the face detection system created by Viola et al. [7] on $24 \times 24$ pixel images collected by crawling the web. They obtained an accuracy of 79 percent. The performance of some well-known human face recognition algorithms are compared in terms of accuracy [8]. Buchala et al. [9] proposed a gender classification system based on a sub set of selected features using principal component analysis (PCA), Self organizing maps (SOM), and curvilinear component analysis (CCA). Best results are obtained using 759 PCA components, about 92.25 percent detection rate. Main disadvantage is over-fitting because, the size of the faces is restricted to $128 \times 128$ pixels and the test set is composed by only little number of faces [9]. Baluja and Rowley [10] proposed an adaboost system for gender classification with manually aligned faces. They evaluated a database of human faces by varying face image scaling, translation, and rotation. An experimental comparison between the Adaboost and SVM classifier is proposed [10]. Most of the previous works use manual detection and alignment of the face images which has been shown to improve performance [11]. Only few studies have automatically extracted faces using a face detector $[2,3,12]$. Some major challenges of gender classification are to account for the effects of illumination, background clutter, pose and etc. Practical systems have to be robust enough to take these issues into consideration. Most of the work in gender classification assumes that the frontal views of faces, which are pre-aligned and free of distracting background clutters, are available [2]. 
Shmaglit and Khryashchev [13] proposed an approach for gender classification based on two steps: feature extraction and classification. First of all, the input image is converted to HSV color space. Next, adaptive feature set is extracted for localize face images. Finally, SVM is used to classify two gender groups. Good localization for face detecting is one of the main advantages. But high computational complexity can be considered as one of the main disadvantages, which reduce its usability in online applications [13]. An image processing descriptor called Local Binary Patterns (LBP) first has been proposed by Ojala et al. [14], to analysis textures. LBP has been used in various vision applications such as face recognition [15], texture classification [16], surface defect detection [17, 18], image retrieval [19] and etc. Shan [20] used local binary patterns as feature descriptor for gender classification. The authors extracted histogram based on primary version of LBP. Lian and Liu [21] used support vector machine as classifier correlated with local binary patterns.

As a new work, Tapia and Perez [22] claim that feature selection may decrease the Complexity of gender classification. In this respect, the mutual information between statistical face features is used to select an optimum subset of more related features. Finally, SVM is used for classification stage. High detection rate in comparison with some methods is one of the main advantages [22]. But feature selection may add a new complexity to the system. So, it cannot be useful in human identification applications as preprocess. Also, selected features may be sensitive to noise, because selected sub set may be different in each dataset.

The primary version of local binary patters which is used as feature extractor is sensitive to zoom, rotate, gray-scale and size $[20,21]$. In this respect, Ojala et al. [16] modified two dimensional version of local binary patters are proposed and its high quality to analysis textures are discussed. The proposed feature extraction algorithm in this paper is based on modified version of local binary patterns. In this respect, proposed approach is categorized in appearance based methods. Also, the nearest neighbor is applied as classifier, which is evaluated using log likelihood ratio as distance metric. In the result part, the detection rate of the proposed approach is compared with some well know methods in this literature. The results show the high quality of the proposed to classify genders accurately. Rotation invariant, gray-scale invariant, size invariant, low sensitivity to noise, and low computational complexity are some other advantages of the proposed approach. All of the advantage is discussed in a clear way separately.

\section{PRPOSED APPROACH \\ GENDER \\ CLASSIFICATION}

Gender classification is categorized as a visual pattern classification problem. Near all of the works in this scope involves 2 main stages: a) Faces or head features b) classifying those features using pre-labeled database. The proposed approach has a main flowchart as shown in Figure 2. As it is shown in the Figure 2, the novelty of the proposed approach is providing local texture information as features using ILBP. Also, the kullback-leibler divergence is evaluated using log likelihood ratio to improve the recognition rate.

The proposed approach decreases the run time of human identification applications in the smart phones. So, the relation between the proposed gender classification approach and human identification App is shown in the Figure 3.

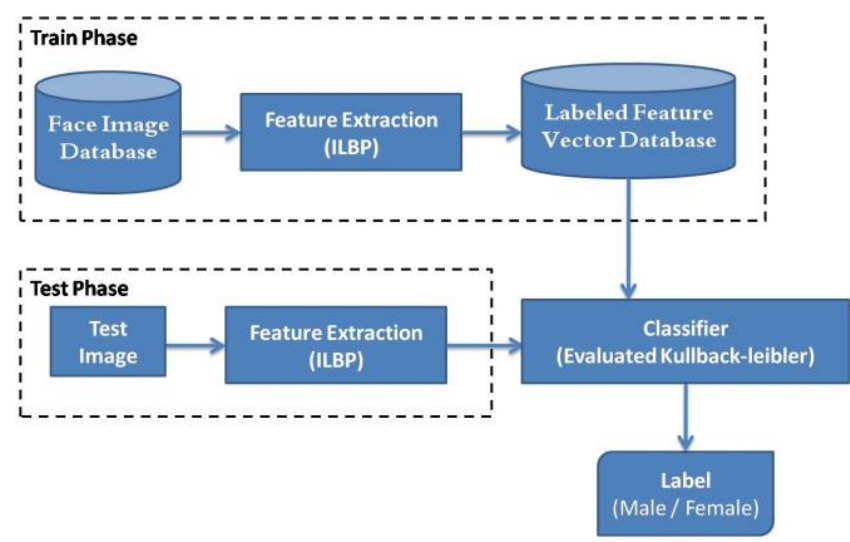

Figure 2. The flowchart of the proposed gender classification system



Figure 3. The flowchart of related between human identification application in smart phones and proposed gender classification

\subsection{Feature extraction step}

Detection rate is much related to the discrimination of extracted features. The main visual difference between the human face images of man and female is usually in the face texture. For example, the body texture of men is usually thicker and has more edges than women. The results of the researchers in previous papers have shown that color and shape information cannot make a significant difference between these two groups. So, in this paper texture information is extracted from human faces using improved local binary patterns (ILBP).

\subsubsection{Basic local binary patterns}

Pietikäinen et al. [14], introduced an image descriptor titled local binary pattern to consider texture information. The LBP is an efficient operator which describes the local contrast and local spatial structure of an image in multi resolutions. Experimental results showed high severability and discriminative power of basic LBP for texture classification [14]. In order to evaluate the LBP, at a given pixel position $\left(\mathrm{x}_{\mathrm{c}}\right.$, $\mathrm{y}_{\mathrm{c}}$ ), LBP is defined as an ordered set of binary intensity comparisons between the center and its surrounding neighbors. In mostly cases, neighborhoods are considered circular because of achieving the rotation invariant. Points which the coordination's are not exactly located at the center of pixel would be found by bilinear interpolation technique. In Figure 
4, some circular neighbors are shown with different radius from $\mathrm{R}=1$ to $\mathrm{R}=2$ and respected different number of neighborhoods pixels (P). Of course, in some cases such as surface defect detection $[17,18]$ the squared neighborhood is considered to reduce computational complexity.



(a)

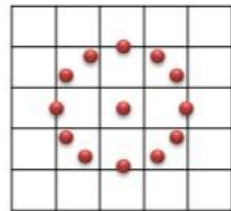

(b)

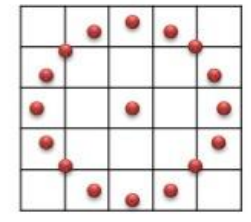

(c)

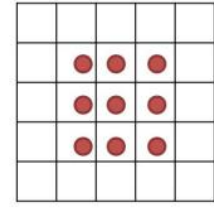

(d)

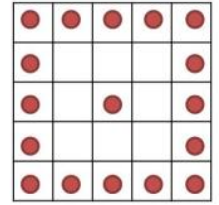

(e)
Figure 4. Some examples of desired neighborhoods in LBP (a) Circular with $\mathrm{P}=8, \mathrm{R}=1$ (b) Circular with $\mathrm{P}=12, \mathrm{R}=1.5$ (c)Circular with $\mathrm{P}=16, \mathrm{R}=2$ (d) Square with $\mathrm{P}=8, \mathrm{R}=1$ (e) Square with $\mathrm{P}=16, \mathrm{R}=2$ (1).

Now, the LBP are defined at desired neighborhood using Eq.

$$
\operatorname{LBP}_{P, R}\left(X_{c}, Y_{c}\right)=\sum_{k=1}^{p} \Omega\left(g_{k}-g_{c}\right) 2^{k-1}
$$

where, " $\mathrm{g}_{\mathrm{c}}$ " corresponds to the grey value of the center pixel and " $g_{k}$ " to the grey values of the $\mathrm{k}^{\text {th }}$ neighborhood pixel. So, $P$ will be the total number of neighborhoods in desired manner, and function $\Omega(\mathrm{x})$ is defined as follows:

$$
\Omega(x)=\left\{\begin{array}{lll}
1 & \text { if } \quad x \geq 0 \\
0 & \text { else }
\end{array}\right.
$$

The $\mathrm{LBP}_{\mathrm{P}, \mathrm{R}}$ descriptor produces $2^{\mathrm{P}}$ different output values, corresponding to the extracted binary patterns.

\subsubsection{Improved local binary patterns}

Low discrimination and high computational complexity are disadvantage of basic LBP which are discussed with details [14]. To solve these problems, Tajeripour and Fekri-Ershad [16] defined a uniformity measure "U" to categorize extracted binary patterns in significant groups. $\mathrm{U}$, corresponds to the number of spatial transitions (bitwise $0 / 1$ changes) in the extracted binary pattern. The computing process of uniformity measure is shown in Eq. (3). For example, patterns 01001100 have $U$ value of 4, while 11000001 have $U$ value of 2 .

$$
\begin{gathered}
U\left(\operatorname{LBP}_{\mathrm{P}, \mathrm{R}}\left(\mathrm{x}_{\mathrm{c}}, \mathrm{y}_{\mathrm{c}}\right)\right)=\left|\Omega\left(\mathrm{g}_{1}-\mathrm{g}_{\mathrm{c}}\right)-\Omega\left(\mathrm{g}_{\mathrm{P}}-\mathrm{g}_{\mathrm{c}}\right)\right|+ \\
\sum_{\mathrm{k}=2}^{\mathrm{P}} \Omega\left(\mathrm{g}_{\mathrm{k}}-\mathrm{g}_{\mathrm{c}}\right)-\Omega\left(\mathrm{g}_{\mathrm{k}-1}-\mathrm{g}_{\mathrm{c}}\right)
\end{gathered}
$$

Next, Patterns are grouped in two classes. Hence, patterns with uniformity amount less than $\mathrm{U}_{\mathrm{T}}$ are categorized as uniform patterns. The patterns with uniformity more than $U_{T}$ classified as non-uniforms. Finally, a label is assigned to each neighborhood using Eq. (4). The total number of ones in extracted binary patterns is considered as label for uniform patterns, and label $\mathrm{P}+1$ is assigned to all of the non uniform patterns.

$$
I L B P_{P, R}^{\text {riu }_{T}}=\left\{\begin{array}{l}
\sum_{k=1}^{P}\left(g_{k}-g_{c}\right) \quad \text { if } U\left(L B P_{P, R}\right) \leq U_{T} \\
P+1 \quad \text { elsewhere }
\end{array}\right.
$$

In notation of $I L B P$ descriptor, the word riu $_{T}$ shows uniform patterns that have $U$ value lower than $U_{T}$. $L_{B} P_{P, R}$ will assign a label from zero to $P$ to all uniform patterns. Also, unique label $P+1$ will be assigned to all of the non-uniform ones. So, uniformity threshold should be optimized that uniform labels cover majority patterns in the image. Experimental results show that if $\mathrm{U}_{\mathrm{T}}$ is chosen $P / 4$, only a small part of the patterns in the texture takes label $\mathrm{P}+1[17,18]$

As it was described, a label is assigned to each neighborhood. Regarding the Eq. (4), if the number of neighbors is considered " $\mathrm{P}$ " pixels, applying $\mathrm{LBP}_{\mathrm{P}, \mathrm{R}}$ will assign a label from zero to "P" to uniform segments and label "P+1" to non-uniform segments. Using uniformity process make ILBP resistant to image rotation and gray scale variations. Image rotation may disturb the position of neighbors in relation with center pixel, but the relation between the neighbors will be same after rotation. A visual example is shown in Figure 5.

\begin{tabular}{|c|c|c|}
\hline 5 & 80 & 85 \\
\hline 94 & 90 & 46 \\
\hline 124 & 23 & 88 \\
\hline
\end{tabular}

(a)

Extracted Pattern: 00011000

$\mathrm{U}=2$

Label $=2$

\begin{tabular}{|c|c|c|}
\hline 124 & 94 & 5 \\
\hline 23 & 90 & 80 \\
\hline 88 & 46 & 85 \\
\hline
\end{tabular}

(c)

Extracted Pattern: 00000110

$\mathrm{U}=2$

Label $=2$

\begin{tabular}{|c|c|c|}
\hline 94 & 5 & 80 \\
\hline 124 & 90 & 85 \\
\hline 23 & 88 & 46 \\
\hline
\end{tabular}

(b)

Extracted Pattern: 00001100

$\mathrm{U}=2$

Label $=2$

\begin{tabular}{|c|c|c|}
\hline 88 & 23 & 124 \\
\hline 46 & 90 & 94 \\
\hline 85 & 80 & 5 \\
\hline
\end{tabular}

(d)

Extracted Pattern: 00000011

$\mathrm{U}=2$

Label $=2$
Figure 5. Rotation invariant proof of ILBP

Applying ILBP extract local texture information based on some predefined label. In order to make it ready for classification, texture information should be converted to a numeric feature vector. So, for every image, a feature vector can be extracted with " $\mathrm{P}+2$ " dimensions. In this respect, first ILBP $_{P, R}$ should applied on the whole image and the labels are assigned to neighbors. Then the occurrence probability of each label in the image is regarded as one of the dimensions of the feature vector. The occurrence probability of a specific label in the image can be approximated by the ratio of the number of that label to the number of all labels (Eq. (5)).

$$
F=<N_{P_{0}} / N_{\text {total }}, N_{P_{1}} / N_{\text {total } 1_{1}}, \ldots, N_{P_{p+1}} / N_{\text {total }}>
$$

where, $N_{P i}$ is the number of neighbors that labeled as $i$, and $N_{\text {total }}$ is the total number of neighbors. The extracted feature vector $(F)$ is probabilistic which means the addition of all dimension values are one. 


\subsection{Classification phase}

Fix and Hodges [23] developed a nonparametric algorithm for pattern classification, which is known as kullback-leibler divergence $(\mathrm{KL})$. It is a lazy classifier that stores all label instances and classifies new sample using a distance/nondistance criteria. An instance is classified by a majority vote of its neighbors, with the instance being assigned to the class most common among its $\mathrm{T}$ nearest samples measured by a distance criteria. Using different distance/similarity criteras is main property of the KL.

For instance, Nguyen et al. [24] used cosine as a distance measure, for face verification. Pearson correlation similarity is proposed for text classification, which provided good accuracy [25]. In information theory, the KL divergence [26], also called discrimination information, is a measure of the difference between two probability distributions $A$ and $B$. It is not symmetric in $A$ and $B$. In applications, $A$ typically represents the true distribution of date observations, while $B$ typically represents a theory or approximation of $A$. Hindi [27] listed more than 50 different similarity and distance metrics in terms of binary, nominal, numeric, etc.

One of main novelties of the proposed approach is using evaluated kullback-leibler as classifier. In this regard, near all of the similarity/distance criteria was performed in $\mathrm{KL}$ divergence [27]. Finally, log likelihood provided highest classification accuracy rate. The extracted vector using ILBP is a probabilistic measure, where the sum of all dimension values is one. The log-likelihood ratio is specialized for probabilistic vectors. Log-likelihood ratio is used for texture classification based on local binary patterns [16, 17]. Log likelihood ratio provides maximum accuracy for surface defect detection using modified local binary patterns [18]. Loglikelihood ratio between two probabilistic feature vectors like $A$ and $B$, is computed as described in the Eq. (6).

$$
L(A, B)=\sum_{k=0}^{P+1} A_{k} \log \left(A_{k} / B_{k}\right)
$$

where, $L$ is the log-likelihood ratio and $A_{F}$ shows the feature vector which is extracted for train image A. Also, $A_{k}$ shows the value of $k^{\text {th }}$ dimension in vector $A$. B shows the feature vector which is extracted for test image $B$. Also, $B_{k}$ shows the value of $\mathrm{k}^{\text {th }}$ dimension in vector $\mathrm{B}$. The KL divergence is a non similarity measure, since minimization of ratio shows the similarity to specific class. In our application, $A$ and $B$ can be considered as extracted feature vectors for two texture images using Eq. (6). Also, in our experiments, T nearest samples to the test image are selected based on KL divergence ratio. Finally, test image is classified by a majority vote of its $\mathrm{T}$ nearest neighbors. It is denoted by $\mathrm{T}-\mathrm{KL}$ in this paper as follows.

\section{EXPERIMENTAL RESULTS}

To achieve a fair performance comparison, the proposed approach was evaluated upon two databases:
I. Self-Collected Database
II. II.ICPR database as follows

\subsection{Comparison results on self-collected database}

In order to evaluate the performance, 200 human face images are selected from wiki dataset [28], in different pose, face orientation, rotation, zoom and size. The above challenges are common challenges that occur when shooting a profile image with a smart phone.

The collected database includes 100 face images of females and 100 images for male. The proposed gender classification system is applied on total database images. Finally, the evaluated T-KL which is discussed in section 3.2 was used to classify genders ( $\mathrm{Two}$ Labels $=$ male $\&$ female) based on $\mathrm{T}=1$, 3,5 . The classification accuracy rate is shown in Table 1 . Hence, some other well-known classifiers such as SVM, Simple KNN, J48 Tree and Naïve Bayes are used for classification as follows. in all cases, 10-fold method is performed as validation method.

Table 1. Proposed gender classification accuracy rate (\%) using different classifiers on self-collected database

\begin{tabular}{cccccc}
\hline Method & 1-KL & 3-KL & 5-KL & SVM & J48 Tree \\
\hline Accuracy & 93.65 & $\mathbf{9 4 . 3 5}$ & 92.67 & 90.83 & 84.22 \\
Method & 1-NN & 3-NN & 5-NN & Naïve Bayes & MLP \\
Accuracy & 88.04 & 90.25 & 86.52 & 79.54 & 91.32 \\
\hline
\end{tabular}

The maximum classification rate is provided using evaluated $\mathrm{T}-\mathrm{KL}$ with $\mathrm{T}=3$. In Table 2 , the proposed gender classification approach is compared with some other state-ofthe-art methods in this literature. Lian et al. [21] proposed an approach based on primary version of LBP and SVM as classifier. Shakhnarovich et al. [6] used adaboost to propose fast and ethnicity gender classification for real time face images. Leng et al. [29], proposed an appearance-based method based on Gabor features and Fuzzy SVM as classifier. Some of the self-collected database images are shown in Figure 6.

Table 2. Proposed gender classification accuracy rate (\%) in comparison with well-known approaches on self collected database

\begin{tabular}{|c|c|c|c|}
\hline Method & Proposed Approach & $\begin{array}{c}\text { LBP + } \\
\text { SVM [21] }\end{array}$ & $\begin{array}{c}\text { Harrlike + } \\
\text { Adaboost [6] }\end{array}$ \\
\hline Accuracy & $\mathbf{9 4 . 1 5}$ & 91.56 & 82.45 \\
\hline Method & $\begin{array}{c}\text { LBPH+Haarlike + } \\
\text { (Adaboost/SVM) [30] }\end{array}$ & $\begin{array}{c}\text { Gabor + } \\
\text { Fuzzy } \\
\text { SVM [29] }\end{array}$ & $\begin{array}{c}\text { Patch Based } \\
+ \text { Bayesian } \\
{[31]}\end{array}$ \\
\hline Accuracy & & 94.27 & 89.35 \\
\hline
\end{tabular}
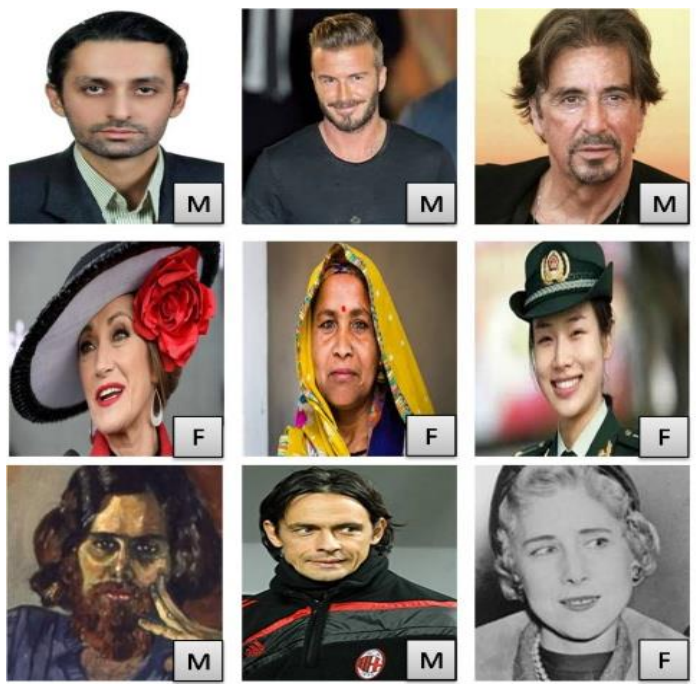

Figure 6. Some of the human face images in self-collected database 


\subsection{Comparison results on ICPR database}

Gender classification near all of the times is performed on human face images. There are many benchmarks to evaluate the performance of face recognition, face detection, gender classification or facial identification methods. ICPR is a stateof-the-art benchmark for face image analysis [32]. Different pose, skin color, illumination variation is some of the ICPR characteristics. In this respect, our proposed approach is applied on ICPR database to evaluate its performance. ICPR consists of 15 sets of images. Each set contains of 2 series of 93 images of the same person at different poses. There are 30 people in the database, having various skin colors. The pose, or head orientation are different in images. Among the images, 16 images are male and 14 images are female. The proposed approach was applied on this database images and detection rate is computed which is shown in Table 3.

Table 3. Proposed gender classification accuracy rate compared with well-known approaches on ICPR database

\begin{tabular}{|c|c|}
\hline Method & $\begin{array}{c}\text { Classification } \\
\text { Accuracy(\%) }\end{array}$ \\
\hline Proposed Approach & $\mathbf{9 4 . 7 6}$ \\
\hline Harrlike + Adaboost [6] & 87.65 \\
\hline LBP + SVM [21] & 90.65 \\
\hline $\begin{array}{c}\text { Mutal informatio+Statistical } \\
\text { features+SVM [22] }\end{array}$ & 94.53 \\
\hline Gabor + FuzzySVM [29] & 94.84 \\
\hline LBPH+Haarlike + SVM [30] & 91.06 \\
\hline Patch Based + Bayesian [31] & 90.83 \\
\hline HCLBP + KNN [33] & 93.67 \\
\hline
\end{tabular}

As it is shown in tables $2 \& 3$, the proposed approach provides more classification accuracy rate than near all of other approaches. Of course, the combination of Gabor and fuzzy SVM provides the best classification rate between them, but the computational complexity of combination Gabor and fuzzy SVM [29] is more than proposed method because of computation Gabor features. The computational complexity of Gabor filtering is compared with local binary patterns [34].

\subsection{Advantages}

According to the results, the proposed approach provides high classification rate. The proposed approach has some more important advantages as follows:

\subsubsection{Low computational complexity}

In image processing cases, one of the main challenges is computational complexity. In order to evaluate the complexity, numbers of required operations are computed such as division, multiplication, addition and subtraction [17, 18, 35-37]. To apply $\mathrm{LBP}_{\mathrm{P}, \mathrm{R}}$, on image, total number of required operations are shown in Table 4 . Where, the size of image is $\mathrm{W} \times \mathrm{W}$.

As, it's shown in Table 4 , the total number of required operations for an image is very low, against other appearancebased methods such as statistical features, Gabor filters, wavelet features. For example, to extract wavelet features from an image in size of $256 \times 256$, more than $14,800,000$ comparison, subtraction and additions are required. But, applying proposed $\mathrm{LBP}_{16,2}$ just need 18 multiplications, 18 divisions, 16 addition, and 1,040,400 comparisons [18].

Table 4. Total Number of required operations for applying $\operatorname{LBP}_{P, R}$

\begin{tabular}{|c|c|c|c|c|}
\hline Operation & Comparison & Multiplication & Division & Addition \\
\hline LBP $_{\mathbf{8}, \mathbf{1}}$ & $(\mathrm{W}-1) \times(\mathrm{W}-1) \times 8$ & 10 & 10 & 8 \\
\hline $\mathbf{L B P}_{\mathbf{1 6}, \mathbf{2}}$ & $(\mathrm{W}-2) \times(\mathrm{W}-2) \times 16$ & 18 & 18 & 16 \\
\hline $\mathbf{L B P}_{\mathbf{2 4 , 3}}$ & $(\mathrm{W}-3) \times(\mathrm{W}-3) \times 24$ & 26 & 26 & 24 \\
\hline $\mathbf{L B P} \mathbf{P}, \mathbf{R}$ & $(\mathrm{W}-\mathrm{R}) \times(\mathrm{W}-\mathrm{R}) \times \mathrm{P}$ & $\mathrm{P}+2$ & $\mathrm{P}+2$ & $\mathrm{P}$ \\
\hline
\end{tabular}

\subsubsection{Rotation invariant}

Some gender classification approaches are sensitive too rotation [38]. The modified version of local binary patterns is rotation invariant $[16,39]$. In Eq. (3), the uniform measure is computed by computing the transition between 0 and 1 . Suppose, if the image rotates, pixel's coordination may be changed, but its neighbors value are fix because of using circular neighbors. In this respect, the number of transitions between 0 and 1, will not change, respectively uniformity measure.

\subsubsection{Scale invariant}

After applying the $\mathrm{LBP}_{\mathrm{P}, \mathrm{R}}$ on each pixel, the occurrence probability of each label in image is computed by dividing to size of image as its shown Eq.(5). In this respect the proposed feature extracted vector (Eq. (6)) is probabilistic and it is not sensitive to size of human face image.

\subsubsection{Low noise sensitivity}

The noise is an unwanted signal. The pixel value may be changed based on noise, so the LBP label for that pixel may not be correct. According to the Eq. (6), each dimension shows occurrence probability of labels in whole face image. So, little changes in the total number of LBP labels may not result big changes in the feature dimension values. In this respect, the proposed approach is low sensitive to noise.

Different affiliations shall be listed in separate lines. Do not insert any punctuation at the end of each affiliation. If all the authors are affiliated to the same organization, type that affiliation just once.

\subsection{Results on parental monitoring over child on well- known social network applications}

As noted, one of the benefits of gender detection algorithms is for parents to analysis the social network contacts of their child in terms of gender. In this regard, the profile images of contacts of four different users in two popular social networks (WhatsApp and FaceTime) were classified by the proposed algorithm. The algorithm presented in this paper identified gender of contacts with 87.54 percent accuracy. The details of this experiment are shown in the table below. 
Table 5. Proposed gender classification accuracy rate (\%) on social network profile images

\begin{tabular}{|c|c|c|c|}
\hline \multicolumn{4}{|c|}{ User \#1 } \\
\hline Gender & Male & Female & Average \\
\hline $\begin{array}{c}\text { Classification } \\
\text { accuracy }\end{array}$ & 86.32 & 90.74 & 88.53 \\
\hline \multicolumn{4}{|c|}{ User \#2 } \\
\hline Gender & Male & Female & Average \\
\hline $\begin{array}{c}\text { Classification } \\
\text { accuracy }\end{array}$ & 87.38 & 89.92 & 88.65 \\
\hline \multicolumn{4}{|c|}{ User \#3 } \\
\hline Gender & Male & Female & Average \\
\hline $\begin{array}{c}\text { Classification } \\
\text { accuracy }\end{array}$ & 90.04 & 91.12 & 90.58 \\
\hline \multicolumn{4}{|c|}{ User \#4 } \\
\hline Gender & Male & Female & Average \\
\hline $\begin{array}{c}\text { Classification } \\
\text { accuracy }\end{array}$ & 84.92 & 88.47 & 86.69 \\
\hline \multicolumn{4}{|c|}{} \\
\hline
\end{tabular}

\subsection{Results on time consuming of human identification applications}

As mentioned, one of the goals of this paper is to speed up the process of human identification applications in smart phones. In order to investigate this, a creative experiment was put forward. First, a human identification application was designed locally based on the Android operating system. Next, it is installed on a Sony Xperia LT25i smart phone. A database of 400 human face images was collected for it. Human identification was performed for 100 images randomly. The results showed that the average identification time for each image took 2.3 seconds. Then the proposed gender classification algorithm was added to the human identification method as a pre-processing step. The results showed that the average identification time for each image may take about 1.9 seconds. Thus, on average, the speed of human identification in the designed application decreased approximately 17.39 percent.

\section{CONCLUSIONS}

The main aim of this paper was to propose a robust approach for gender classification in smartphone applications. In order to achieve the aim, an appearance-based method is proposed included two steps. First, improved local binary patterns are used to extract local texture information. Next, an evaluated version of k-nearest neighbors is proposed which uses $\log$ likelihood ratio as distance measure. In the result part, gender classification is done using evaluated T-KL on two benchmark databases, self-collected and ICPR. The proposed approach provides classification accuracy about 94.76 on ICPR dataset. The classification accuracy is compared with some other stateof-art methods. Results show that the performance of the proposed method is higher than state-of-the-art methods in the related area.

One of the unique benefits of this paper is to increase the quality of parental monitoring over their children's contacts on social networks. Experimental results on user profile images in two social networks, WhatsApp and FaceTime showed that the proposed method can accurately classify the gender of contact with accuracy 87.54 percent.

Increasing the speed of human identification apps on smart phones is another advantage of this method. The results of an Android-based human identity app showed that using the proposed gender classification algorithm can reduce the run time of it about 17.39 percent.

Proposed approach provides some other advantages such as rotation invariant, size invariant, low computational complexity, low sensitivity to noise and gray-scale invariant. These advantages claim the robustness of the proposed gender classification approach.

\section{REFERENCES}

[1] Rahman, M.H., Chowdhury, S., Bashar, M.A. (2013). An automatic face detection and gender classification from color images using support vector machine. Journal of Emerging Trends in Computing and Information Science, 4(1): 5-11. https://doi.org/10.1109/iciev.2013.6572576

[2] Briones, A.G., Villarrubia, G., De-Paz, J.F., Corchado, J.M. (2018). A multi-agent system for the classification of gender and age form images. Computer Vision and Image Understanding, 172: 98-106. https://doi.org/10.1016/j.cviu.2018.01.012

[3] Moghaddam, B., Yang, M.H. (2002). Learning gender with support faces. IEEE Transactions on Pattern Analysis and Machine Intelligence, 24(5): 707-711. https://doi.org/10.1109/34.1000244

[4] Matta, F., Saeed, U., Mallauran, C., Dugelay, J.L. (2008). Facial gender recognition using multiple sources of visual information. In: Workshop on Multimedia Signal Processing, Cairns, Qld, Australia, pp. 785-790. https://doi.org/10.1109/mmsp.2008.4665181

[5] Yang, M.H., Moghaddam, B. (2000). Gender classification with support vector machines. In: International Conference of Automatic Face and Gesture Recognition, Grenoble, France, France, pp. 306-311. https://doi.org/10.1109/afgr.2000.840651

[6] Shakhnarovich, G., Viola, A.P., Moghaddam, B. (2002). A unified learning framework for real time face detection and classification. Proceedings of Fifth IEEE International Conference on Automatic Face Gesture Recognition, Washington, DC, USA. https://doi.org/10.1109/AFGR.2002.1004124

[7] Viola, P., Jones, M. (2001). Rapid object detection using a boosted cascade of simple features. In: IEEE Computer Society Conference on Computer Vision and Pattern Recognition, Kauai, HI, USA, pp. 511-518. https://doi.org/10.1109/cvpr.2001.990517

[8] Castrillon-Santana, M., Vuong, Q.C. (2007). An analysis of automatic gender classification. In: Conference on Progress in Pattern Recognition, Image Analysis, and Applications, pp. 271-280. https://doi.org/10.1007/9783-540-76725-1_29

[9] Buchala, S., Davey, N., Frank, R.J., Gale, T.M., Loomes M., Kanargard, W. (2010). Gender classification of face images: The role of global and feature-based information. Lecture Notes in Computer Science, 3316: 763-768. https://doi.org/10.1007/978-3-540-30499-9_117

[10] Baluja, S., Rowley, H.A. (2007). Boosting sex identification performance. International Journal of Computer Vision, 71(1): 111-119. https://doi.org/10.1007/s11263-006-8910-9

[11] Makinen, E., Raisamo, R. (2008). An experimental comparison of gender classification methods. Pattern 
Recognition Letters, 29(10): 1544-1556. https://doi.org/10.1016/j.patrec.2008.03.016

[12] Gutta, S., Wechsler, H., Phillips, P.J. (1998). Gender and ethnic classification of face images. In: Third IEEE International Conference on Automatic Face and Gesture Recognition, Nara, Japan, pp. 194-199. https://doi.org/10.1109/afgr.1998.670948

[13] Shmaglit, L., Khryashchev, V. (2013). Gender classification of human face images based on adaptive features and support vector machines. Optical Memory and Neural Networks, 22(4): 228-235. https://doi.org/10.3103/s1060992x13040036

[14] Pietikäinen, M., Ojala, T., Xu, Z. (2000). Rotationinvariant texture classification using feature distributions. Pattern Recognition, 33(1): 43-52. https://doi.org/10.1016/s0031-3203(99)00032-1

[15] Ahonen, T., Hadid, A., Pietikainen, M. (2006). Face description with local binary patterns: application to face recognition. IEEE Transactions on Pattern Analysis and Machine Intelligence, 28(12): 2037-2041. https://doi.org/10.1109/tpami.2006.244

[16] Ojala, T., Pietikainen, M., Maenpaa, T. (2002). Multiresolution gray-scale and rotation invariant texture classification with local binary patterns. IEEE Transaction on Pattern Analysis and Machine Intelligence, 24(7): 971-987. https://doi.org/10.1109/tpami.2002.1017623

[17] Tajeripour, F., Fekri-Ershad, S. (2014). Developing a novel approach for stone porosity computing using modified local binary patterns and single scale retinex. Arabian Journal for Science and Engineering, 39(2): 875889. https://doi.org/10.1007/s13369-013-0725-8

[18] Fekri-Ershad, S., Tajeripour, F. (2012). A robust approach for surface defect detection based on one dimensional local binary patterns. Indian Journal of Science and Technology, 5(8): 3197-3203. https://doi.org/10.1080/08839514.2017.1378012

[19] Takala, V., Ahonen, T., Pietikäinen, M. (2005). Blockbased methods for image retrieval using local binary patterns. Image Analysis Lecture Note in Computer Science, 3540 : $882-891$ https://doi.org/10.1007/11499145_89

[20] Shan, C.F. (2012). Learning local binary patterns for gender classification on real world face images. Pattern Recognition Letters, 33(4): 431-437. https://doi.org/10.1016/j.patrec.2011.05.016

[21] Lian, H.C., Lu, B.L. (2006). Multi view gender classification using local binary patterns and support vector machine. Advances in Neural Networks: Lecture Notes in Computer Science, 3972: 202-209. https://doi.org/10.1007/11760023_30

[22] Tapia, J.E., Perez, C.A. (2019). Clusters of features using complementary information applied to gender classification from face images. IEEE Access, 7: 7937479387. https://doi.org/10.1109/access.2019.2923626

[23] Fix, E., Hodges, J.L. (1951). Discriminatory analysis nonparametric discrimination: Consistency properties. Technical Report 4, USAF School of Aviation Medicine, Randolph Field, Texas, USA https://doi.org/10.1037/e471672008-001

[24] Nguyen, H., Bai, L. (2010). Cosine similarity metric learning for face verification. Proc. of 10th Asian Conf. on Computer Vision, Queenstown, New Zealand, pp.
709-720. https://doi.org/10.1007/978-3-642-19309-5_55

[25] Yin, C., Shi, L., Wang, J. (2017). Short text classification technology based on KNN+Hierarchy SVM. Lecture Notesin Electrical Engineering, pp. 633-639. https://doi.org/10.1007/978-981-10-5041-1_100

[26] Kullback, S., Leibler, R.A. (1951). On information and sufficiency. Ann. Math. Stat., 22(1): 79-86. https://doi.org/10.1214/aoms/1177729694

[27] Hindi, K.E. (2013). Specific-class distance measures for nominal attributes. AI Communications, 26(3): 261-279. https://doi.org/10.3233/aic-130565

[28] Wiki Dataset, https://data.vision.ee.ethz.ch /cvl/rrothe/ imdb-wiki/, accessed on July. 5, 2019.

[29] Leng, X.M., Wang, Y.D. (2008). Improving generalization for gender classification. In: 15th IEEE International Conference on Image Processing, pp. 16561659. https://doi.org/10.1109/icip.2008.4712090

[30] Mäkinen, E., Raisamo, R. (2008). An experimental comparison of gender classification methods. Pattern Recognition Letters, 29(10): 1544-1556. https://doi.org/10.1016/j.patrec.2008.03.016

[31] Aghajanian, J., Warrell, J., Prince, S.J., Rohn, J.L., Baum B. (2009). Patch-based within object classification. In: IEEE 12th International Conference on Computer Vision, pp. $1125-1132$ https://doi.org/10.1109/iccv.2009.5459352

[32] ICPR dataset, http://wwwprima.inrialpes.fr/Pointing04/data-face.html/, accessed on July 5, 2019.

[33] Fekri-Ershad, S., Tajeripour, F. (2017). Color texture classification based on proposed impulse-noise resistant color local binary patterns and significant points selection algorithm. Sensor Review, 37(1): 33-42. https://doi.org/10.1108/sr-07-2016-0120

[34] Tajeripour, F., Kabir, E., Sheikhi, A. (2008). Fabric defect detection using modified local binary patterns. EURASIP Journal on Advances in Signal Processing, 8: 1-12. https://doi.org/10.1155/2008/783898

[35] Alareqi, M., Rachid, E., Laamari, H. (2006). High level FPGA modeling for image processing algorithms using Xilinx generator. International Journal of Computer Science and Telecommunications, 5(6): 1-8.

[36] Rocher, R., Menard, D., Scarlet, P., Sentieys, O. (2012). Analytical approach for numerical accuracy estimation of fixed points systems based on smooth operations. IEEE Transactions on Circuits and Systems, 59(10): 2326-2339. https://doi.org/10.1109/tcsi.2012.2188938

[37] Tamura, S., Kawai, H., Mitsumoto, H. (1996). Male/female identification from $8 \times 6$ very low resolution face images by neural network. Pattern Recognition, 29(2): 331-335. $\quad$ https://doi.org/10.1016/00313203(95)00073-9

[38] Golomb, B.A., Lawrence, D.T., Sejnowski, T.J. (1991). SEXNET: A neural network identifies sex from human faces. Advances in Neural Information Processing Systems, pp. 572-577. https://doi.org/10.1007/978-14757-2379-3_3

[39] Tajeripour, F., Saberi, M., Rezaei, M., Fekri-Ershad, S. (2011). Texture classification approach based on combination of random threshold vector technique and co-occurrence matrixes. In Proc. of Int. Conf. on Computer Science and Network Technology, pp. 23032306. https://doi.org/10.1109/iccsnt.2011.6182434 